\title{
ON THE WEISS CONJECTURE FOR FINITE LOCALLY PRIMITIVE GRAPHS
}

\author{
MARSTON D. CONDER ${ }^{1}$, CAI HENG LI ${ }^{2}$ AND CHERYL E. PRAEGER ${ }^{2}$ \\ ${ }^{1}$ Department of Mathematics, University of Auckland, Auckland, New Zealand \\ ${ }^{2}$ Department of Mathematics, University of Western Australia, \\ Nedlands, WA 6907, Australia
}

(Received 31 March 1998)

\begin{abstract}
A graph $\Gamma$ is said to be locally primitive if, for each vertex $\alpha$, the stabilizer in Aut $\Gamma$ of $\alpha$ induces a primitive permutation group on the set of vertices adjacent to $\alpha$. In 1978, Richard Weiss conjectured that for a finite vertex-transitive locally primitive graph $\Gamma$, the number of automorphisms fixing a given vertex is bounded above by some function of the valency of $\Gamma$. In this paper we prove that the conjecture is true for finite non-bipartite graphs provided that it is true in the case in which Aut $\Gamma$ contains a locally primitive subgroup that is almost simple.
\end{abstract}

Keywords: vertex-transitive graphs; locally primitive graphs; quasiprimitive permutation groups; almost simple groups

AMS 1991 Mathematics subject classification: Primary 05025

\section{Preamble}

In 1978 Richard Weiss [21] conjectured that, for a finite vertex-transitive locally primitive graph $\Gamma$, the number of automorphisms fixing a given vertex is bounded above by some function of the valency of $\Gamma$. This conjecture is an analogue of the Conjecture of Charles Sims [13], that for a finite primitive permutation group with a suborbit of length $d>2$, the order of a point stabilizer is bounded above by a function of $d$. Although Sims' Conjecture has been proved true [1], the truth of Weiss's Conjecture is still unsettled. In this paper we prove that the Weiss Conjecture is true for finite non-bipartite graphs provided that it is true for graphs that admit almost simple locally primitive groups of automorphisms.

A graph $\Gamma$ is said to be locally primitive if, for every vertex $\alpha$, the stabilizer of $\alpha$ induces a primitive permutation group on the set of vertices adjacent to $\alpha$. In the special case of 2-arc transitive graphs, that is, the case where the permutation group induced by a vertex stabilizer is not only primitive but is 2-transitive, the Weiss Conjecture has been proved as a culmination of a long sequence of papers by several authors: namely, Gardiner [4-6], Weiss [22-24] and Trofimov [16-19]. Trofimov and Weiss [20] have 
tried to extend this result to the locally primitive case under the condition that a vertex stabilizer induces a primitive group (abstractly) isomorphic to $\operatorname{PSL}(n, q)$ on the set of adjacent vertices; a complete solution is still not available even for this special case. From these results of Trofimov and Weiss, it is easily shown that the Weiss Conjecture is true for graphs of prime valency and for graphs of valency at most 20 . We give the details in Proposition 4.1. However, it seems unlikely that the truth of the Weiss Conjecture will be determined by a consideration of all possible local actions.

The strategy used in the proof of the Sims Conjecture was to apply the O'Nan-Scott Theorem for finite primitive permutation groups to reduce the problem to the case where the primitive group $G$ is almost simple, that is $S \leqslant G \leqslant \operatorname{Aut}(S)$ for some non-abelian simple group $S$. A result of Thompson [15] showed that a vertex stabilizer has a normal $p$-subgroup the index of which is bounded above by a function of the size $d$ of the suborbit. This result, combined with explicit information about the various families of finite simple groups, led to a complete proof of the Sims Conjecture. Thus the proof depends on the classification of the finite simple groups.

Unfortunately, there seems to be no reduction possible for Weiss's Conjecture to the case of finite vertex primitive graphs. However, for finite non-bipartite graphs, a reduction for Weiss's Conjecture was obtained by the third author $[8, \S 3]$ to the case where the locally primitive group of automorphisms is quasiprimitive on vertices, that is, every nontrivial normal subgroup is transitive on vertices. In this paper, we apply the O'Nan-Scott Theorem for finite quasiprimitive permutation groups proved in [9] to reduce the proof of Weiss's Conjecture for non-bipartite graphs to the case where the locally primitive group of automorphisms is almost simple. We discuss some of the difficulties that are encountered in the almost simple case in $\S 3$, and we end with some remarks on the consequences of the work of Weiss and Trofimov [18-21] in $\S 4$.

Let $\Gamma$ be a finite connected graph, and let $G$ be a subgroup of Aut $\Gamma$, the automorphism group of $\Gamma$. For a vertex $\alpha$ of $\Gamma$, let $\Gamma(\alpha)$ denote the set of vertices adjacent to $\alpha$ in $\Gamma$. Then $\Gamma$ is said to be $G$-locally primitive if, for all vertices $\alpha, G_{\alpha}$ induces a primitive permutation group $G_{\alpha}^{\Gamma(\alpha)}$ on $\Gamma(\alpha)$. For non-bipartite graphs $\Gamma$ it is easy to show that, if $\Gamma$ is $G$-locally primitive, then $G$ acts transitively on the vertices of $\Gamma$. Thus, for nonbipartite graphs, Weiss's Conjecture is the following.

Weiss Conjecture for non-bipartite graphs (see [21]). There exists a function $f: \mathbb{N} \rightarrow \mathbb{N}$ such that, if $\Gamma$ is a finite, non-bipartite, $G$-locally primitive graph of valency $d$, then for a vertex $\alpha,\left|G_{\alpha}\right| \leqslant f(d)$.

We may also state this conjecture in the quasiprimitive almost simple case as follows. The socle $\operatorname{soc}(G)$ of a group $G$ is the product of its minimal normal subgroups, and a finite group is said to be almost simple if its socle is a non-abelian simple group.

Almost simple Weiss Conjecture. There is a function $f: \mathbb{N} \rightarrow \mathbb{N}$ such that, if $\Gamma$ is a finite $G$-locally primitive graph of valency $d$ for some almost simple group $G$ and $\operatorname{soc}(G)$ is vertex-transitive, then for a vertex $\alpha,\left|G_{\alpha}\right| \leqslant f(d)$. 
Our reduction theorem may thus be stated as follows.

Main Theorem. The Weiss Conjecture is true for all finite non-bipartite graphs if and only if the almost simple Weiss Conjecture is true.

\section{Preliminaries}

First we prove a simple property regarding the primes that divide $|H|$, where $H$ is a subgroup of automorphisms of a connected graph and $H$ fixes a vertex. We denote the vertex set of a graph $\Gamma$ by $V \Gamma$ and the edge set by $E \Gamma$.

Lemma 1.1. Let $\Gamma$ be a connected graph of valency $d$. Suppose that $G$ is a group of automorphisms of $\Gamma$ which acts transitively on $V \Gamma$. Then

(i) for $\alpha \in V \Gamma$, each prime divisor of $\left|G_{\alpha}\right|$ divides $\left|G_{\alpha}^{\Gamma(\alpha)}\right|$; and

(ii) for an edge $\{\alpha, \beta\}$ of $\Gamma$, each prime divisor of $\left|G_{\alpha \beta}\right|$ is less than $d$.

Proof. Let $p$ be a prime divisor of $\left|G_{\alpha}\right|$, and let $g$ be an element of $G_{\alpha}$ of order $p$. Then there exists $\alpha^{\prime} \in V \Gamma$ that is not fixed by $g$. Since $\Gamma$ is connected, there is a path from $\alpha$ to $\alpha^{\prime}: \alpha=\alpha_{0}, \alpha_{1}, \ldots, \alpha_{l}=\alpha^{\prime}$. There exists some $k<l$ such that $\alpha_{i}^{g}=\alpha_{i}$ for all $i$ with $0 \leqslant i \leqslant k$, and $\alpha_{k+1}^{g} \neq \alpha_{k+1}$. Thus $g \in G_{\alpha_{k}}$, and, since $\alpha_{k+1} \in \Gamma\left(\alpha_{k}\right), \alpha_{k+1}^{g} \in \Gamma\left(\alpha_{k}\right)$. Let $g^{*}$ be the element of $G_{\alpha_{k}}^{\Gamma\left(\alpha_{k}\right)}$ induced by $g$. Then $g^{*} \in G_{\alpha_{k}}^{\Gamma\left(\alpha_{k}\right)}$ and $\alpha_{k+1}^{g^{*}} \neq \alpha_{k+1}$. It follows that $o\left(g^{*}\right)=p$, so $p$ divides $\left|G_{\alpha_{k}}^{\Gamma\left(\alpha_{k}\right)}\right|=\left|G_{\alpha}^{\Gamma(\alpha)}\right|$.

Now assume that $p$ is a prime divisor of $\left|G_{\alpha \beta}\right|$, and let $g$ be an element of $G_{\alpha \beta}$ of order $p$. Arguing as in the previous paragraph, there exists $k \geqslant 1$ and a path $\alpha=$ $\alpha_{0}, \beta=\alpha_{1}, \ldots, \alpha_{k}, \alpha_{k+1}$, such that $\alpha_{i}^{g}=\alpha_{i}$ for all $i \in\{0, \ldots, k\}$ and $\alpha_{k+1}^{g} \neq \alpha_{k+1}$. Thus $g \in G_{\alpha_{k-1} \alpha_{k}}$, and, since $\alpha_{k+1} \in \Gamma\left(\alpha_{k}\right)$ and $\alpha_{k+1} \neq \alpha_{k-1}, \alpha_{k+1}^{g} \in \Gamma\left(\alpha_{k}\right) \backslash\left\{\alpha_{k-1}\right\}$. Let $g^{*}$ be the element of $G_{\alpha_{k-1} \alpha_{k}}^{\Gamma\left(\alpha_{k}\right)}$ induced by $g$. Then $g^{*} \in G_{\alpha_{k-1} \alpha_{k}}^{\Gamma\left(\alpha_{k}\right)}$ and $\alpha_{k+1}^{g^{*}} \neq \alpha_{k+1}$. It follows that $o\left(g^{*}\right)=p$, so $p$ divides $\left|G_{\alpha_{k-1} \alpha_{k}}^{\Gamma\left(\alpha_{k}\right)}\right|$. As the orbits of $G_{\alpha_{k-1} \alpha_{k}}^{\Gamma\left(\alpha_{k}\right)}$ are of size at most $d-1$, we have $p \leqslant d-1$.

A subgroup $H$ of a group $G$ is said to be core-free in $G$ if $\bigcap_{g \in G} H^{g}=1$. For a finite group $G$ and a core-free subgroup $H$ of $G$, we can construct connected graphs $\Gamma$ such that $G \leqslant$ Aut $\Gamma$ and $G$ is arc-transitive as follows (see [12]).

Definition 1.2. Let $G$ be a finite group. Suppose that there are a core-free subgroup $H<G$ and an element $g \in G$ such that $\langle H, g\rangle=G$ and $g^{2} \in H$. A graph $\Gamma=\Gamma(G, H, g)$ is defined by $V \Gamma=\{H x \mid x \in G\}$ with $\{H x, H y\}$ an edge of $\Gamma$ if and only if $x y^{-1} \in H g H$.

Such a graph has the following properties.

Lemma 1.3 (see [12]). Let $\Gamma=\Gamma(G, H, g)$ be a graph defined as in Definition 1.2. Then $G$, acting by right multiplication, is a subgroup of Aut $\Gamma$ and $G_{\alpha}=H$, where 
$\alpha$ is the vertex $H$ of $\Gamma$. Moreover, $\Gamma$ is a connected $G$-arc-transitive graph of valency $\left|H: H \cap H^{g}\right|$.

Conversely, if $G$ acts faithfully and arc-transitively on a finite connected graph $\Gamma$, then $\Gamma \cong \Gamma(G, H, g)$ for some core-free subgroup $H \leqslant G$ and some $g \in G$ as in Definition 1.2.

A graph $\Sigma$ is called a quotient graph of a graph $\Gamma$ if there is a surjective map $\rho$ from $V \Gamma$ to $V \Sigma$ such that $\rho$ preserves the adjacency relation, that is $\rho$ induces a surjection from $E \Gamma$ to $E \Sigma$. It easily follows that if $K$ is a subgroup of $G$ containing $H,\langle H, g\rangle=G$ and $g^{2} \in H$, then $\Gamma(G, K, g)$ is a quotient graph of $\Gamma(G, H, g)$.

A permutation group $G$ on a set $\Omega$ is said to be regular if $G$ is transitive on $\Omega$ and $G_{\alpha}=1$ for every $\alpha \in \Omega$.

Lemma 1.4 (see [7, Lemma 2.1]). Let $\Gamma$ be a connected graph and suppose that $G \leqslant$ Aut $\Gamma$ is transitive on $V \Gamma$. Suppose that $N$ is a normal subgroup of $G$ which is regular on $V \Gamma$. Then $G=N \rtimes G_{\alpha}$ and $G_{\alpha}$ is faithful on $\Gamma(\alpha)$.

Lemma 1.5 (see [2, Theorem 4.2A]). Let $G$ be a transitive group on $\Omega$, and let $C_{\mathrm{Sym}(\Omega)}(G)$ be the centralizer of $G$ in $\operatorname{Sym}(\Omega)$. If $C_{\mathrm{Sym}(\Omega)}(G)$ is transitive on $\Omega$, then $G$ is regular on $\Omega$.

For a non-negative integer $i$, let $\Gamma_{i}(\alpha)$ be the set of vertices of $\Gamma$ that are at distance at most $i$ from $\alpha$, and let $G_{i}(\alpha)$ be the kernel of $G_{\alpha}$ on $\Gamma_{i}(\alpha)$, that is, the subgroup of $G_{\alpha}$ that fixes every vertex in $\Gamma_{i}(\alpha)$. Clearly, $G_{i}(\alpha)$ is normal in $G_{\alpha}$, and further we have the following lemma.

Lemma 1.6. Let $\Gamma=\Gamma(G, H, g)$ be a graph defined as in Definition 1.2, and let $\alpha$ be the vertex $H$ of $\Gamma$ and $\beta=\alpha^{g}$. Then, for any positive integer $i$,

(i) $G_{i+1}(\alpha) \leqslant G_{i}(\alpha) \cap G_{i}(\beta)$; and

(ii) $g$ normalizes $G_{i}(\alpha) \cap G_{i}(\beta)$.

Proof. Since $\beta \in \Gamma(\alpha)$, if $\gamma \in \Gamma_{i}(\beta)$, then $\gamma \in \Gamma_{j}(\alpha)$ for $j=i-1, i$ or $i+1$. Thus $\Gamma_{i}(\alpha) \cup \Gamma_{i}(\beta) \leqslant \Gamma_{i+1}(\alpha)$, so $G_{i+1}(\alpha) \leqslant G_{i}(\alpha) \cap G_{i}(\beta)$ as in part (i).

For any $x \in G_{i}(\alpha)^{g}, x=g^{-1} y g$ for some $y \in G_{i}(\alpha)$, so $\beta^{x}=\beta^{g^{-1} y g}=\alpha^{y g}=\alpha^{g}=\beta$, that is, $x \in G_{i}(\beta)$. It follows that $G_{i}(\alpha)^{g}=G_{i}(\beta)$. Since $g^{2} \in G_{\alpha}$, we have that $\alpha=\alpha^{g^{2}}=$ $\beta^{g}$ and it follows that $G_{i}(\beta)^{g}=G_{i}(\alpha)$. Therefore, $\left(G_{i}(\alpha) \cap G_{i}(\beta)\right)^{g}=G_{i}(\alpha)^{g} \cap G_{i}(\beta)^{g}=$ $G_{i}(\beta) \cap G_{i}(\alpha)$ as in part (ii).

In $\S 2$ we shall consider quasiprimitive permutation groups $G$, that is subgroups $G \leqslant$ $\operatorname{Sym}(\Omega)$ such that all non-trivial normal subgroups of $G$ are transitive on $\Omega$. For the proof of the Main Theorem we shall need the next lemma about the action on a $G$ quasiprimitive $G$-locally primitive graph $\Gamma$ of a normal subgroup $N$ of $G$.

Proposition 1.7. Suppose that $G$ is quasiprimitive on the vertex set of a finite connected graph $\Gamma$ and that $G_{\alpha}^{\Gamma(\alpha)}$ is primitive, for $\alpha \in V \Gamma$. Let $N$ be a non-trivial normal subgroup of $G$. If $N_{e}(\alpha)=1$ for some integer $e$, then $G_{e+1}(\alpha)=1$. 
Proof. Since $G$ is quasiprimitive, $N$ is transitive on $V \Gamma$. If $N$ is regular on $V \Gamma$, then the result follows from Lemma 1.4. Thus we may assume that $N$ is not regular on $V \Gamma$. So $N_{\alpha} \neq 1$ and, since $\Gamma$ is connected, it follows that $N_{\alpha}^{\Gamma(\alpha)} \neq 1$. Since $N_{\alpha}^{\Gamma(\alpha)}$ is a normal subgroup of the primitive group $G_{\alpha}^{\Gamma(\alpha)}, N_{\alpha}$ is transitive on $\Gamma(\alpha)$. Hence $\Gamma$ is $N$-arc-transitive, so, by Lemma $1.3, \Gamma \cong \Gamma\left(N, N_{\alpha}, g\right)$ for some $g \in N$ such that $\left\langle N_{\alpha}, g\right\rangle=N$. If $G_{e}(\alpha)=1$, the result is true so we may assume that $G_{e}(\alpha) \neq 1$. Now $N \cap G_{e}(\alpha)=N_{e}(\alpha)=1$. The normal subgroups $N_{\alpha}$ and $G_{e}(\alpha)$ of $G_{\alpha}$ normalize each other and so centralize each other. Let $\beta:=\alpha^{g}$. By Lemma $1.6, G_{e+1}(\alpha) \leqslant G_{e}(\alpha) \cap G_{e}(\beta)$ and $g$ normalizes $G_{e}(\alpha) \cap G_{e}(\beta)$. Since $N_{\alpha}$ centralizes $G_{e}(\alpha), N_{\alpha}$ centralizes $G_{e}(\alpha) \cap G_{e}(\beta)$, so $N=\left\langle N_{\alpha}, g\right\rangle$ normalizes $G_{e}(\alpha) \cap G_{e}(\beta)$. Since $N \cap\left(G_{e}(\alpha) \cap G_{e}(\beta)\right) \leqslant N \cap G_{e}(\alpha)=1$ and $N \triangleleft G$, it follows that $N$ centralizes $G_{e}(\alpha) \cap G_{e}(\beta)$. However, since $N$ is transitive but not regular on $V \Gamma$, by Lemma $1.5, C_{G}(N)$ is not transitive on $V \Gamma$. Since $G$ is quasiprimitive on $V \Gamma$, the intransitive normal subgroup $C_{G}(N)$ must be trivial. Thus $G_{e}(\alpha) \cap G_{e}(\beta) \leqslant C_{G}(N)=1$, so $G_{e+1}(\alpha) \leqslant G_{e}(\alpha) \cap G_{e}(\beta)=1$ as required.

\section{Proof of the Main Theorem}

Let $\Gamma$ be a finite connected non-bipartite arc-transitive graph of valency $d>2$, and let $G$ be a subgroup of Aut $\Gamma$ that is transitive on $V \Gamma$, and such that for $\alpha \in V \Gamma, G_{\alpha}$ is primitive on $\Gamma(\alpha)$. To prove the Main Theorem, by Praeger $[8, \S 3]$, we may assume that $G$ is quasiprimitive on $V \Gamma$. Let $N$ be the socle of $G$. Our first result deals with the case where $N$ has a regular normal subgroup.

Proposition 2.1. If $N$ has a normal subgroup that is regular on $V \Gamma$, then $\left|G_{\alpha}\right|$ is bounded by a function of $d$; in fact $\left|G_{\alpha}\right| \leqslant d !((d-1) !)^{d}$.

Proof. Since $G$ is quasiprimitive on $V \Gamma, N$ is transitive on $V \Gamma$. Further, $N_{\alpha} \triangleleft G_{\alpha}$, and, since $G_{\alpha}$ is primitive on $\Gamma(\alpha)$, if $N_{\alpha} \neq 1$, then $N_{\alpha}$ is transitive on $\Gamma(\alpha)$. Thus either $N$ is regular on $V \Gamma$ or $\Gamma$ is an $N$-arc-transitive graph. If $G$ has a regular normal subgroup $N$, then, by Lemma $1.4, G=N \rtimes G_{\alpha}$ and $G_{\alpha}$ is faithful on $\Gamma(\alpha)$. Therefore, $G_{\alpha} \leqslant \mathrm{S}_{d}$ and $\left|G_{\alpha}\right| \leqslant d$ !. Thus we may assume that $G$ has no regular normal subgroup. In particular, $N$ is not regular on $V \Gamma$, so, by Lemma 1.3, $\Gamma=\Gamma\left(N, N_{\alpha}, g\right)$ for some 2-element $g \in N$ such that $g^{2} \in N_{\alpha}$ and $N=\left\langle N_{\alpha}, g\right\rangle$.

Suppose that $N$ has a normal subgroup $L$ that is regular on $V \Gamma$. By Lemma 1.4, $N=L \rtimes N_{\alpha}$ and $N_{\alpha}$ is faithful on $\Gamma(\alpha)$. Thus $N_{1}(\alpha)=1$, and hence, by Proposition 1.7, $G_{2}(\alpha)=1$. Thus $G_{\alpha}$ is faithful on $\Gamma_{2}(\alpha)$, so $\left|G_{\alpha}\right| \leqslant d !((d-1) !)^{d}$.

From the O'Nan-Scott Theorem for finite quasiprimitive permutation groups in [9], one of the following holds: $N$ has a regular normal subgroup, or $N$ is a non-abelian simple group (so $G$ is almost simple), or $G$ has type PA. The third type PA is described as follows (see [11]). The socle $N=T_{1} \times \cdots \times T_{l} \cong T^{l}$ for some finite non-abelian simple group $T$ and integer $l \geqslant 2$. There is a proper subgroup $R$ of $T$ such that $N_{\alpha}$ is a subdirect product of $R^{l}$, that is, $N_{\alpha}$ projects onto each of the direct factors $R$. This means that 
$G_{\alpha}<L=G_{\alpha} R^{l}<G$ so that there is a $G$-invariant partition $\Sigma$ of $V \Gamma$ such that $L$ is the setwise stabilizer of the block $\sigma$ of $\Sigma$ containing $\alpha$. Moreover, the action of $G$ on $\Sigma$ is faithful and is permutationally isomorphic to the product action of $G$ on a set $\Delta^{l}$. We may identify $\Sigma$ with $\Delta^{l}$ and we have $G \leqslant W=H$ wr $\mathrm{S}_{l}$, where $H \leqslant \operatorname{Sym}(\Delta)$ and $H$ is an almost simple group with socle $T$ which is quasiprimitive on $\Delta, N$ is the socle of $W$, and $W$ acts on $\Sigma$ in product action.

Our next result deals with quasiprimitive groups of type PA under the assumption that the almost simple Weiss Conjecture is true. Because we consider three different graphs in the proof of this proposition, we shall use a refinement of the notation $G_{e}(\alpha)$ for a subgroup $G \leqslant$ Aut $\Gamma$ fixing $\Gamma_{e}(\alpha)$ pointwise, namely, we shall write $G_{e}(\alpha)=G_{e}(\alpha ; \Gamma)$.

Proposition 2.2. Suppose that $G$ is of type PA (as in [9] or [1 1]). Assume that the almost simple Weiss Conjecture is true. Then $\left|G_{\alpha}\right|$ is bounded by a function of $d$.

Proof. We have $N=T_{1} \times \cdots \times T_{l} \cong T^{l}$, where each $T_{i} \cong T$ is a non-abelian simple group, and $l \geqslant 2$. Moreover, there is a $G$-invariant partition $\Sigma$ of $V \Gamma$ such that the action of $G$ on $\Sigma$ is faithful and is permutationally isomorphic to the product action of $G$ on a set $\Delta^{l}$. As above, we identify $\Sigma$ with $\Delta^{l}$ and we have $G \leqslant W=H$ wr S $\mathrm{S}_{l}$, where $H \leqslant \operatorname{Sym}(\Delta)$ and $H$ is an almost simple group with socle $T$ which is quasiprimitive on $\Delta$, $N$ is the socle of $W$, and $W$ acts on $\Sigma$ in product action. So, for $\sigma=(\delta, \ldots, \delta) \in \Sigma=\Delta^{l}$, $W_{\sigma}=H_{\delta} \mathrm{wr} \mathrm{S}_{l}$. Moreover, setting $R:=H_{\delta} \cap T$, if $\alpha \in V \Gamma$ belongs to the 'block' $\sigma$ of $\Sigma$, then it was proved in [9] that $N_{\alpha}$ is a subdirect product of $N_{\sigma}=R^{l}$, that is $N_{\alpha}$ projects onto each of the $l$ direct factors $R$ of $N_{\sigma}$.

We complete the proof by showing that there exists a positive integer $e$ that is bounded by a function of $d$ such that $N_{e}(\alpha ; \Gamma)=1$, using the assumption that the almost simple Weiss Conjecture is true. Note that $G$ permutes $\left\{T_{1}, \ldots, T_{l}\right\}$ transitively by conjugation since $G$ is quasiprimitive. Further, if $G_{1}$ is the subgroup of index $l$ in $G$ that normalizes $T_{1}$, then $G_{1}$ induces a subgroup of $\operatorname{Sym}(\Delta)$ in its action on the first coordinates of elements of $\Sigma$; this subgroup is almost simple with socle $T$ and we take $H$ to be this subgroup. In particular, since $N$ is transitive on $\Sigma$, it follows that the subgroup $\left(G_{1}\right)_{\sigma}$ induces the group $H_{\delta}$ in its action on the first coordinates of elements of $\Sigma$.

As above, $\sigma=(\delta, \ldots, \delta)$ and $\alpha \in \sigma$. We have $N_{\sigma}=R_{1} \times \cdots \times R_{l}=R^{l}$, where $R_{i}=\left(T_{i}\right)_{\delta} \cong R$. Let $\Gamma_{\Sigma}$ be the quotient graph of $\Gamma$ corresponding to the partition $\Sigma$ of $V \Gamma$, and let $\Gamma_{\Sigma}(\sigma)$ denote the set of neighbours of $\sigma$ in $\Gamma_{\Sigma}$. Let $\beta \in \Gamma(\alpha)$ and $\eta=$ $\left(\delta_{1}, \ldots, \delta_{l}\right) \in \Sigma$ such that $\beta \in \eta$. Then $\eta \in \Gamma_{\Sigma}(\sigma)$. Define $\Phi_{i}(\delta)=\delta_{i}^{R_{i}}$ and $\Psi_{i}(\delta)=\delta_{i}^{H_{\delta}}$, the orbits of $R_{i}$ and $H_{\delta}$, respectively, containing $\delta_{i}$, for $i=1, \ldots, l$. We claim that

$$
\Gamma_{\Sigma}(\sigma)=\eta^{R^{l}}=\delta_{1}^{R_{1}} \times \cdots \times \delta_{l}^{R_{l}}=\Phi_{1}(\delta) \times \cdots \times \Phi_{l}(\delta) .
$$

Note that $\left(G_{\sigma}\right)_{1}=G_{1} \cap G_{\sigma}$ induces the permutation group $H_{\delta}$ in its action on the first coordinates of elements of $\Sigma$. Thus each $\delta_{1}^{\prime}$ in $\Psi_{1}(\delta)$ occurs as the first entry of some $\eta^{\prime} \in \Gamma_{\Sigma}(\sigma)$. Since $G=N G_{\sigma}, G_{\sigma}$ permutes the $T_{i}$ transitively and it follows that each $\delta_{i}^{\prime} \in \Psi_{i}(\delta)$ occurs as the $i$ th entry of some element of $\Gamma_{\Sigma}(\sigma)$. Hence $\Gamma_{\Sigma}(\sigma) \subseteq \prod \Psi_{i}(\sigma)$ and $\Gamma_{\Sigma}(\sigma)$ projects onto each $\Psi_{i}(\sigma)$. 
Now $\Gamma(\alpha) \cap \eta$ contains the vertex $\beta$ and is a block of imprimitivity for the primitive action of $G_{\alpha}$ on $\Gamma(\alpha)$. If $\Gamma(\alpha) \subseteq \eta$, then $\Gamma(\beta) \subseteq \sigma$ and it follows by connectivity that $V \Gamma \subseteq \sigma \cup \eta$, which is not the case. It follows that $\Gamma(\alpha) \cap \eta=\{\beta\}$. Similarly, $\left|\Gamma(\alpha) \cap \eta^{\prime}\right| \leqslant 1$ for each $\eta^{\prime} \in \Gamma_{\Sigma}(\sigma)$. Note that $N_{\alpha}$ is transitive on $\Gamma(\alpha)$. Thus the subset of $\Gamma_{\Sigma}(\sigma)$ consisting of those 'blocks' of $\Sigma$ that contain vertices of $\Gamma(\alpha)$ is precisely $\eta^{N_{\alpha}}$. Now $\eta^{N_{\alpha}} \subseteq \eta^{N_{\sigma}}$, and, as $N_{\sigma}=R_{1} \times \cdots \times R_{l}$, we have $\eta^{N_{\sigma}}=\delta_{1}^{R_{1}} \times \cdots \times \delta_{l}^{R_{l}}=\Phi_{1}(\delta) \times \cdots \times \Phi_{l}(\delta)$. Moreover, since $N_{\sigma}$ is transitive on $\sigma$ (for $\sigma$ is a block of imprimitivity for the transitive group $N$ on $V \Gamma)$, for each $\alpha^{\prime} \in \sigma$ there is an element $x \in N_{\sigma}$ such that $\alpha^{x}=\alpha^{\prime}$. It follows that the subset of $\Gamma_{\Sigma}(\sigma)$ that contains vertices of $\Gamma\left(\alpha^{\prime}\right)$ is $\left(\eta^{N_{\alpha}}\right)^{x}=\eta^{N_{\alpha} x} \subseteq \eta^{N_{\sigma}}=\prod \Phi_{i}(\delta)$. Since this is true for each $\alpha^{\prime} \in \sigma$, we have $\Gamma_{\Sigma}(\sigma) \subseteq \prod \Phi_{i}(\delta) \subseteq \prod \Psi_{i}(\delta)$, and since $\Gamma_{\Sigma}(\sigma)$ projects onto each $\Psi_{i}(\delta)$, we have $\Phi_{i}(\delta)=\Psi_{i}(\delta)$ for each $i$. Thus each $\Phi_{i}(\delta)$ is an $H_{\delta}$-orbit. Since $\Gamma_{\Sigma}(\sigma) \supseteq \eta^{N_{\sigma}}=\prod \Phi_{i}(\alpha)$, we have $\Gamma_{\Sigma}(\sigma)=\prod \Phi_{i}(\delta)$ as claimed.

Note that $G_{\sigma}$ is transitive on $\{1,2, \ldots, l\}$, namely, for each $i$, there exists

$$
c:=\left(h_{1}, \ldots, h_{l}\right) g_{0} \in G_{\sigma} \leqslant H_{\delta} \mathrm{wrS}_{l}
$$

such that $g_{0}$ maps 1 to $i$. Then $\Gamma_{\Sigma}(\sigma)$ contains

$$
\eta^{c}=\left(\delta_{1 g_{0}^{-1}}^{h g_{0}^{-1}}, \ldots, \delta_{l g_{0}^{-1}}^{h_{l g^{-1}}^{-1}}\right)
$$

which has $i$ th entry

$$
\delta_{i g_{0}^{-1}}^{h_{i g_{0}^{-1}}}=\delta_{1}^{h_{1}} \in \Phi_{1}(\delta) .
$$

So $\Phi_{i}(\delta)=\Phi_{1}(\delta)$. Set $\Phi(\delta):=\Phi_{1}(\delta)=\cdots=\Phi_{l}(\delta)$. Then $\Gamma_{\Sigma}(\sigma)=\Phi(\delta)^{l}$.

We claim that $H_{\delta}$ acts primitively on $\Phi(\delta)$. If $\psi(\delta):=\left\{B^{h} \mid h \in H_{\delta}\right\}$ is a non-trivial block system of $H_{\delta}^{\Phi(\delta)}$, then $\psi(\delta)^{l}$ is a partition of $\Sigma$ invariant under $H_{\delta} \mathrm{wr} \mathrm{S}_{l}$ and, hence, is preserved by $G_{\sigma}$. Hence, if $\tilde{B}=B_{1} \times \cdots \times B_{l}$ is the block of $\psi(\delta)^{l}$ containing $\eta$, then $\tilde{B}^{G_{\alpha}}$ is a partition of $\eta^{G_{\alpha}}$ preserved by $G_{\alpha}$ (and note that each member of $\tilde{B}^{G_{\alpha}}$ is a subset of $\left.\Phi(\delta)^{l}\right)$. Since the permutation groups induced by $G_{\alpha}$ on $\eta^{G_{\alpha}}$ and on $\Gamma(\alpha)$ are permutationally isomorphic, $G_{\alpha}$ is primitive on $\eta^{G_{\alpha}}$. Hence, $\tilde{B}^{G_{\alpha}}$ is a trivial block system for $G_{\alpha}$ in $\eta^{G_{\alpha}}$. However, $1<\left|B_{1}\right|<|\Phi(\delta)|$ and $\eta^{G_{\alpha}}$ projects onto $\Phi(\delta)$ in the first coordinate, so $\tilde{B}$ is a non-trivial block, which is a contradiction. Hence $H_{\delta}^{\Phi(\delta)}$ is primitive. Let $d_{0}=|\Phi(\delta)|$. Then $d_{0} \leqslant d$. Let $\Phi$ denote the $H$-arc-transitive graph with vertex set $\Delta$ and vertex-stabilizer $H_{\delta}$ corresponding to the suborbit $\Phi(\delta)$. Then, from our assumption that the almost simple Weiss Conjecture holds, we have $\left|H_{\delta}\right| \leqslant f_{\mathrm{AS}}\left(d_{0}\right)$ for some function $f_{\mathrm{AS}}$ on $\mathbb{N}$. Since $d_{0} \leqslant d$, there exists a positive integer $e$ that is bounded by a function of $d$ such that $H_{e}(\delta ; \Phi)=1$. For any $x \in N_{e}\left(\sigma ; \Gamma_{\Sigma}\right)$, we may write $x=h_{1} h_{2} \ldots h_{l}$ such that $h_{i} \in H$. Since $\Gamma_{\Sigma}(\sigma)=\Phi(\sigma)^{l}$, we have $h_{i} \in H_{e}(\delta ; \Phi)$, so $h_{i}=1$ and, hence, $x=1$. Therefore, $N_{e}\left(\sigma ; \Gamma_{\Sigma}\right)=1$. Since $\Gamma_{\Sigma}$ is a quotient graph of $\Gamma$, we have $N_{e}(\alpha ; \Gamma) \leqslant N_{e}\left(\sigma ; \Gamma_{\Sigma}\right)=1$. By Proposition 1.7, $G_{e+1}(\alpha ; \Gamma)=1$ and so $\left|G_{\alpha}\right|$ is bounded by a function of $d$.

Note that $d \geqslant|\Phi(\delta)|^{l}=d_{0}^{l}$. Thus our proof shows that $\left|G_{\alpha}\right| \leqslant\left|H_{\delta}\right|^{l} l ! \leqslant\left(f_{\mathrm{AS}}\left(d_{0}\right)\right)^{l} l ! \leqslant$ $f_{\mathrm{AS}}\left(d^{1 / l}\right)^{l} l$ !, where $l \leqslant \log _{2} d$. Now we are ready to prove the main theorem. 
Proof of the Main Theorem. Recall that $G$ is quasiprimitive on $V \Gamma$ so that its socle $N$ is transitive on $V \Gamma$. If $N$ has a normal subgroup that is regular on $V \Gamma$, then, by Proposition 2.1, the Main Theorem holds. Thus assume that $N$ does not have a normal regular subgroup on $V \Gamma$. From the O'Nan-Scott Theorem for quasiprimitive groups proved in [9], we easily conclude that $G$ is of type PA or $G$ is almost simple. Therefore, by Proposition 2.2, the theorem holds.

\section{Almost simple case}

In this section we use some examples to discuss the almost simple Weiss Conjecture. We still use $\Gamma$ to denote a finite connected non-bipartite graph of valency $d>2$, and let $G$ be a subgroup of Aut $\Gamma$ that is transitive on $V \Gamma$, and such that for $\alpha \in V \Gamma, G_{\alpha}$ is primitive on $\Gamma(\alpha)$. We also assume that $\Gamma=\Gamma\left(G, G_{\alpha}, g\right)$ for some 2-element $g$ (in the sense of Definition 1.2 and Lemma 1.3), and let $\beta=\alpha^{g}$.

Weiss [23] proved his conjecture for a special class of local actions, namely the case where $G_{\alpha}^{\Gamma(\alpha)}$ is primitive with a regular normal elementary abelian subgroup. Such primitive groups are called affine primitive groups.

Theorem 3.1 (Weiss). If $G_{\alpha}^{\Gamma(\alpha)}$ is an affine primitive group, then $\left|G_{\alpha}\right|$ is bounded by a function of $d$.

This result helps to simplify the proof of the Weiss Conjecture for some classes of almost simple groups $G$.

Proposition 3.2. Suppose that $\operatorname{soc}(G)=\operatorname{PSL}(2, q)$ for some prime power $q \geqslant 4$. Then the Weiss Conjecture is true.

Proof. By Theorem 3.1, if $G_{\alpha}$ is soluble, then $\left|G_{\alpha}\right|$ is bounded by a function of $d$. Thus we assume that $G_{\alpha}$ is insoluble. It follows from Dickson's classification of subgroups of $\operatorname{PSL}(2, q)$ (see $\left[14\right.$, p. 417]) that $\operatorname{PSL}(2, q) \cap G_{\alpha} \cong \operatorname{PSL}\left(2, q_{0}\right)$ or $\operatorname{PGL}\left(2, q_{0}\right)$, where $q_{0}=p^{e_{0}}$ and $e_{0} \mid e$, or $\operatorname{PSL}(2, q) \cap G_{\alpha} \cong A_{5}$. Thus $G_{\alpha}$ is faithful on $\Gamma(\alpha)$, and so $\left|G_{\alpha}\right| \leqslant d !$.

Clearly, a similar argument can be used for some other classes of almost simple groups, in particular for the almost simple Lie-type groups of Lie rank one. However, we do not think that it can easily be extended for arbitrary almost simple groups. In particular, we have been unable to prove the conjecture in the case where $G$ is $\mathrm{A}_{n}$ or $\mathrm{S}_{n}$. We end with a discussion of this family of groups.

Example 3.3. Let $G=\mathrm{A}_{n}$ or $\mathrm{S}_{n}$, which naturally acts on $X=\{1,2, \ldots, n\}$. Then the possibilities for $G_{\alpha}$ can be divided into three classes:

(i) $G_{\alpha}$ is intransitive on $X$;

(ii) $G_{\alpha}$ is transitive on $X$ but has a non-trivial intransitive normal subgroup; and 
(iii) $G_{\alpha}$ is quasiprimitive on $X$.

Let $K=G_{2}(\alpha)$. By [3, Corollary 2.3], $K$ is a $p$-group for some prime $p<d$. If $K=1$, then $G_{\alpha} \cong G_{\alpha}^{\Gamma_{2}(\alpha)}$, so $\left|G_{\alpha}\right|$ is bounded by a function of $d$. Thus we may assume that $K \neq 1$. Then $K$ is a non-trivial proper normal subgroup of $G_{\alpha}$.

This observation is sufficient to prove the Weiss Conjecture for stabilizers in case (iii), that is, for $G_{\alpha}$ quasiprimitive on $X$. It follows from the O'Nan-Scott Theorem in [9] that $K=\mathbb{Z}_{p}^{a}$ and $G_{\alpha} / K \leqslant \mathrm{GL}(a, p)$ for some $a \geqslant 1$. Now, $G_{\alpha} / K$ induces a faithful permutation group on $\Gamma_{2}(\alpha)$ so that $\left|G_{\alpha} / K\right| \leqslant f(d)$ for some function $f$. On the other hand, as $G_{\alpha}$ is primitive on $X$, the $\left(G_{\alpha} / K\right)$-orbit containing a non-zero element of $K$ contains a basis $\left\{x_{1}, x_{2}, \ldots, x_{a}\right\}$ of $K$. Thus, $a \leqslant\left|G_{\alpha} / K\right| \leqslant f(d)$. Since, by Lemma $1.1, p$ divides $\left|G_{\alpha}^{\Gamma(\alpha)}\right|$, we have $p<d$. Therefore, $\left|G_{\alpha}\right|=|K|\left|G_{\alpha} / K\right| \leqslant d^{f(d)} f(d)$.

\section{Some remarks}

Here we discuss some consequences of the work of Weiss and Trofimov [16-24] for the Weiss Conjecture.

Proposition 4.1. The Weiss Conjecture is true for the cases in which the valency of graphs is a prime or is at most 20.

Proof. Let $\Gamma$ be a $G$-locally primitive graph of valency $d$. Then $G_{\alpha}^{\Gamma(\alpha)}$ is a primitive permutation group of degree $d$.

If $G_{\alpha}^{\Gamma(\alpha)}$ is an affine primitive group, then $\left|G_{\alpha}\right|$ is bounded by a function of $d$ by Theorem 3.1. Thus we may assume that $G_{\alpha}^{\Gamma(\alpha)}$ is not affine. In particular $d \geqslant 5$.

Suppose next that $d$ is a prime. Then, by Burnside's Theorem [2, Theorem 3.5B], $G_{\alpha}^{\Gamma(\alpha)}$ is 2-transitive and the proposition follows from $[18,19]$.

Suppose now that $d \leqslant 20$ and that $d$ is not a prime. Then, by [2, Appendix B], $G_{\alpha}^{\Gamma(\alpha)}$ is 2-transitive, or is almost simple with socle $\operatorname{PSL}(n, q)$ for some integers $n \neq 5$ and $q$. By [18-21], $\left|G_{\alpha}\right|$ is bounded by a function of $d$.

These arguments can be extended relatively easily to larger values of the valency $d$. If $d=21$, the only primitive group $G_{\alpha}^{\Gamma(\alpha)}$ that is neither affine nor 2-transitive is $G_{\alpha}^{\Gamma(\alpha)}=\mathrm{A}_{7}$ or $\mathrm{S}_{7}$ on pairs. Similar arguments establish the conjecture for $d=22,23$ and 24 , and for all primitive local actions of degree 25 except primitive groups $G_{\alpha}^{\Gamma(\alpha)}$ in product action with socle $\mathrm{A}_{5} \times \mathrm{A}_{5}$.

Acknowledgements. This work is in part supported by an ARC large grant and the NZ Marsden Fund.

\section{References}

1. P. J. Cameron, C. E. Praeger, J. Saxl and G. M. Seitz, On the Sims conjecture and distance transitive graphs, Bull. Lond. Math. Soc. 15 (1983), 499-506. 
2. J. D. Dixon And B. Mortimer, Permutation groups (Springer, New York and Berlin, 1996).

3. T. Gardiner, Arc transitivity in graphs, Q. J. Math. Oxford (2) 24 (1973), 399-407.

4. T. Gardiner, Doubly primitive vertex stabilizers in graphs, Math. Z. 135 (1974), 157166.

5. T. Gardiner, Arc transitively in graphs, II, Q. J. Math. Oxford (2) 25 (1974), 163-167.

6. T. GaRdiner, Arc transitively in graphs, III, Q. J. Math. Oxford (2) 27 (1976), 313-323.

7. C. D. GoDsil, On the full automorphism group of a graph, Combinatorica 1 (1981), 243-256.

8. C. E. Praeger, Imprimitive symmetric graphs, Ars Combin. 19A (1985), 149-163.

9. C. E. Praeger, An O'Nan-Scott Theorem for finite quasiprimitive permutation groups and an application to 2-arc transitive graphs, J. Lond. Math. Soc. (2) 47 (1993), 227-239.

10. C. E. PRAEger, On a reduction theorem for finite, bipartite, 2-arc transitive graphs, Australas. J. Combin. 7 (1993), 21-36.

11. C. E. PRAEger, Finite quasiprimitive graphs, in Surveys in combinatorics, London Mathematical Society Lecture Note Series, vol. 24 (1997), pp. 65-85.

12. G. SABIDUSSI, Vertex-transitive graphs, Monatsh. Math. 68 (1964), 426-438.

13. C. C. Sims, Graphs and finite permutation groups, Math. Z. 95 (1967), 76-86.

14. M. SUzUKI, Group theory, I (Springer, New York, 1982).

15. J. G. Thompson, Bounds for the orders of maximal subgroups, J. Algebra 14 (1970), $135-138$.

16. V. I. TROFImov, Stabilizers of the vertices of graphs with projective suborbits, Soviet Math. Dokl. 42 (1991), 825-828.

17. V. I. Trofimov, Graphs with projective suborbits (in Russian), Izv. Akad. Nauk SSSR Ser. Mat. 55 (1991), 890-916.

18. V. I. TROFIMOV, Graphs with projective suborbits, cases of small characteristics, I (in Russian), Izv. Akad. Nauk SSSR Ser. Mat. 58 (1994), 124-171.

19. V. I. Trofimov, Graphs with projective suborbits, cases of small characteristics, II (in Russian), Izv. Akad. Nauk SSSR Ser. Mat. 58 (1994), 137-156.

20. V. I. TROFIMOV AND R. WEISS, Graphs with a locally linear group of automorphisms, Math. Proc. Camb. Phil. Soc. 118 (1995), 191-206.

21. R. Weiss, s-transitive graphs, Colloq. Math. Soc. János Bolyai 25 (1978), 827-847.

22. R. WEISS, Groups with a $(B, N)$-pair and locally transitive graphs, Nagoya Math. J. 74 (1979), 1-21.

23. R. WEISS, An application of $p$-factorization methods to symmetric graphs, Math. Proc. Camb. Phil. Soc. 85 (1979), 43-48.

24. R. WeIss, Permutation groups with projective unitary subconstituents, Proc. Am. Math. Soc. 78 (1980), 157-161. 